\title{
Pflegebedürftige freuen sich über bessere Leistungen
}

— Die Begutachtung und Einstufung von Patienten in Pflegegrade soll besser und individueller werden. Dafür gilt seit dem 1. Januar 2017 ein neuer Pflegebedürftigkeitsbegriff, wie er im Zweiten Pflegestärkungsgesetz (PSG II) geschaffen wurde. Die größte Neuerung ist, dass die Pflegesituation von Menschen mit geistigen und seelischen Beeinträchtigungen - etwa einer demenziellen Erkrankung - nun genau so berücksichtigt wie jene von körperlich Beeinträchtigten. In nunmehr fünf Pflegegraden soll die individuelle Situation besser abgebildet werden. Für den neuen Pflegegrad 1 kommen viele Patienten infrage, die bisher noch keinen Zugang zu Pflegeleistungen hatten.

\section{MMW-KOMMENTAR}

Die Neuerung hat auch Auswirkungen auf die Verordnung von Krankenfahrten zur ambulanten Behandlung. Diese sind nur für Patienten mit dem Pflegegrad 3, 4 oder 5 (bisher: Pflegestufe 2 oder 3) zulässig. Auch muss eine dauerhafte Einschränkung der

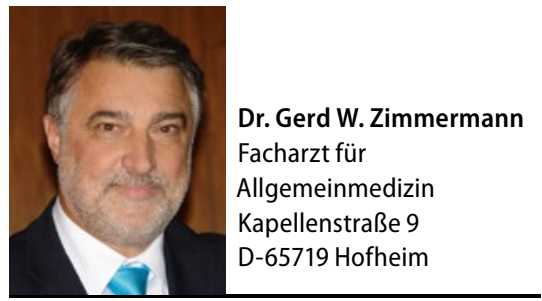

Mobilität vorliegen. Bis das entsprechende Formular angepasst ist, muss allerdings weiterhin das Feld "Pflegestufe 2 bzw. 3 vorgelegt" angekreuzt werden.

Das neue Gesetz hat zur Konsequenz, dass rund 2,7 Millionen Pflegebedürftigen automatisch ein Pflegegrad zuerkannt wird. Menschen mit körperlichen Beeinträchtigungen werden von ihrer Pflegestufe in den nächsthöheren Pflegegrad übergeleitet. Wenn bei ihnen eine dauerhaft erhebliche Einschränkung der Alltagskompetenz vorliegt, gelangen sie sogar in den übernächsten Pflegegrad. Niemand wird also niedrigere Pflegeleistungen beziehen - und viele $\mathrm{Pa}$ tienten sogar höhere.

Damit sollen die spürbaren Leistungsverbesserungen, die bereits zum 1. Januar 2015 eingeführt wurden, weiter ausgeweitet werden. Durch eine Anhebung derPflegebeiträge stehen 2017 rund 6,2 Milliarden Euro zusätzlich zur Verfügung. Der Bedarf an weiteren Beitragsanpassungen wird von nun an alle drei Jahre geprüft.

\section{Unterlagen nun direkt an MDK schicken}

_ Unterlagen für gutachterliche Stellungnahmen bei GKV-Versicherten müssen seit Jahresbeginn direkt an den Medizinischen Dienst der Krankenkassen (MDK) übermittelt werden. Datenschützer hatten das alte Verfahren mit dem Umweg über die Krankenkasse als zu unsicher kritisiert, was schließlich zu dieser Änderung geführt hat.

\section{MMW-KOMMENTAR}

Die Kassen müssen nun für die Übermittlung der Befunde einen vollständig ausgefüllten Weiterleitungsbogen (Muster 86) vorlegen. Dieser enthält u. a. die Anschrift des MDK, eine Vorgangsnummer und die Daten des Patienten, damit die korrekte Zuordnung sichergestellt ist. Auch ein Freiumschlag für den Versand muss dabei sein. Ab dem 1. April
2017 ist hier das Format $C 5$ vorgeschrieben. Die Praxis muss also lediglich die angeforderten Unterlagen in Kopie beilegen und das Ganze direkt an den MDK schicken. Die Kopien können nach Nr. 40144 EBM je Seite berechnet werden.

Ist der Umschlag zu klein, kommen die Versandpauschalen nach den Nrn. 40122 40126 zum Ansatz. 